\section{RMD Open}

Rheumatic \&

Musculoskeletal Diseases

\title{
Missing data in randomised controlled trials of rheumatoid arthritis drug therapy are substantial and handled inappropriately
}

\author{
Nasim A Khan, ${ }^{1}$ Karina D Torralba, ${ }^{2}$ Fawad Aslam (D) ${ }^{3}$
}

\begin{abstract}
To cite: Khan NA, Torralba KD, Aslam F. Missing data in randomised controlled trials of rheumatoid arthritis drug therapy are substantial and handled inappropriately. RMD Open 2021;7:e001708. doi:10.1136/ rmdopen-2021-001708
\end{abstract}

- Additional supplemental material is published online only. To view, please visit the journal online (http://dx.doi.org/10. 1136/rmdopen-2021-001708).

For 'Presented at statement' see end of article.

Received 21 April 2021 Accepted 11 July 2021
Check for updates

(C) Author(s) (or their employer(s)) 2021. Re-use permitted under CC BY-NC. No commercial re-use. See rights and permissions. Published by BMJ.

For numbered affiliations see end of article.

Correspondence to Dr Fawad Aslam; fawadaslam2@gmail.com

\section{ABSTRACT}

Objectives To analyse the amount, reporting and handling of missing data, approach to intention-to-treat (ITT) principle application and sensitivity analysis utilisation in randomised clinical trials (RCTs) of rheumatoid arthritis (RA). To assess the trend in such reporting 10 years apart (2006 and 2016).

Methods Parallel group drug therapy RA RCTs with a clinical primary endpoint.

Results 176 studies enrolling a median of 160 (IQR 62-339) patients were eligible. In terms of actual analysis: $81(46 \%)$ RCTs conducted ITT, 42 (23.9\%) conducted modified ITT while $53(30.1 \%)$ conducted non-ITT analysis. Only 58 of 97 (59.8\%) RCTs reporting an ITT analysis actually performed it. The median (IQR) numbers of participants completing the trial and included in analysis for primary outcome were $86 \%(74 \%-91 \%)$ and $100 \%$ (97.1\%-100\%), respectively. 53 (32.7\%) and 65 (40.1\%) RCTs had $>20 \%$ and $10 \%-20 \%$ missing primary outcome data, respectively. Missing data handling was unreported by 58 of 171 (33.9\%) RCTs. When reported, vast majority used simple imputation methods. No significant trend towards improved reporting was seen between 2006 and 2016. Sensitivity analysis numerically improved from 2006 to $2016(14.7 \%$ vs $21.4 \%)$.

Conclusions There is significant discrepancy in the reported and the actual performed analysis in RA drug therapy RCTs. Nearly one-third of RCTs had $>20 \%$ missing data. The reporting and methods of missing data handling remain inadequate with high usage of non-preferred simple imputation methods. Sensitivity analysis utilisation was low. No trend towards better missing data reporting and handling was seen.

\section{INTRODUCTION}

Randomised controlled trial (RCT) is the gold standard for comparing treatments and determining efficacy. Randomization reduces bias by balancing distribution of known and unknown confounding factors. ${ }^{1}$ The intention-to-treat (ITT) principle preserves randomisation by evaluating all participants as original randomly assigned, regardless of

\section{Key messages}

What is already known about this subject?

- Missing data are common in randomised controlled trials (RCTs).

- Handling of missing data has implication for the results of RCTs.

- Little is known about missing data reporting and its handling in RCTs of rheumatoid arthritis (RA) treatment.

What does this study add?

- Missing data are a significant issue in RCTs in RA.

- More importantly, missing data handling relies heavily on error-prone methods of simple imputation with no trend of improvement.

- Sensitivity analysis usage remains very low.

How might this impact on clinical practice or future developments?

- Increased awareness and requirement of using the preferred imputation methods is needed.

- Journals should require reporting of these methods as well as sensitivity analysis.

deviations from randomised treatment and actual interventions. ${ }^{1-3}$

Most trials have missing data due to protocol deviation, non-adherence, trial noncompletion and other issues. ${ }^{3}$ The ideal analysis, a true ITT analysis, has no missing data. ${ }^{2}$ Per-protocol (PP) or complete-case (CC) analysis include only subjects who adhere to the assigned treatment and complete the study. These approaches generally introduce bias. Missing data undermine the RCT by introducing confounding, unbalancing baseline characteristics and compromising the internal validity. ${ }^{45}$ Moreover, compliant subjects do better than non-compliant ones, irrespective of active treatment or placebo status. ${ }^{6}$ Thus, restricting analysis to those who completed a trial as PP can lead to misleading 
estimates of treatment efficacy. ${ }^{7}$ Non-ITT methods may simply reflect confounding from differences of demographics, prognostic features or subject characteristics. ${ }^{5}$ The ITT approach reflects the real-world settings where subjects drop out, switch treatments or are noncompliant. Although ITT may underestimate the true difference, via dilution, it still represents the best unbiased estimate. ${ }^{9}{ }^{10}$ However, it must be noted that ITT analysis only matters if there are missing data, otherwise ITT and CC are similar.

The term ITT is often used incorrectly ${ }^{11} 12$ as RCTs with post-randomisation exclusions are still described as using 'ITT analysis'. ${ }^{13}$ The term modified ITT (mITT) reflects analyses where participants are excluded for different reason(s) post-randomisation. ${ }^{14}$ mITT analysis usage has increased. ${ }^{15}$ Consequently, the Consolidated Standards of Reporting Trials (CONSORT) statement requires a clear description of subject randomisation and analysis rather than simply stating the method. ${ }^{1}$

Missing data may seriously compromise inferences ascertained from RCTs, ${ }^{16} 17$ limit the ability to draw conclusions ${ }^{18}$ or lead to incorrect inferences about drug safety. ${ }^{19}{ }^{20}$ Missing data can be handled by imputation. Simple imputation (SI) methods include last observation carried forward (LOCF), baseline observation carried forward, non-response imputation (NRI) or poor response imputation. SI assumes lack of temporal change, underestimates true data variability and biases treatment effect estimates. ${ }^{16} 1721$ Multiple imputation, maximum likelihood-based methods such as expectation-maximisation algorithm, or equation-based methods such as full information maximum likelihood or mixed model regression for longitudinal data are generally preferred. These methods incorporate auxiliary information about the missing data and report SE and p values. ${ }^{16}{ }^{1721}$ Any attempt at replacing missing data makes unverifiable assumptions about the distribution of the unobserved or missing data. ${ }^{22}{ }^{23}$ To test these assumption(s), sensitivity analyses with a different assumption(s) should be performed. ${ }^{16172124}$

Studies across disciplines have reported inappropriate use of the ITT principle, incorrect handling of missing data, utilisation of weak imputation methods and lack of sensitivity analyses. ${ }^{13}$ 24-27 Studies in rheumatoid arthritis (RA) exploring this have either been restricted to top medical journals or have also included subjects without RA. ${ }^{25} 27$ Our study examines RA publications in all medical journals and gives temporal trends in missing data reporting and handling, before and after the publication of 2010 CONSORT statement. ${ }^{1}$ Our goal was to identify areas of improvement and create awareness about such shortcomings.

\section{METHODS}

Literature search and RCT selection

MEDLINE and Cochrane Central Register of Controlled Trials databases were searched using terms "rheumatoid arthritis" and "arthritis, rheumatoid" with limits of "Clinical Trials" \& years "2002-3", "2006-7", "2010-11" and "2016". Inclusion criteria were original report of a single RCT in a population with RA, parallel design, drug therapy intervention and a clinical primary endpoint. Non-English language, abstract only, non-clinical endpoint, open-label extensions, population without RA, phase I studies and non-drug intervention studies were excluded. Studies with safety as the primary outcome were also excluded as safety analyses typically focus on the subgroup of trial participants who received the intervention, although such approach may be misleading by ignoring the reasons trial participants stop treatment. ${ }^{28}$

\section{Trial selection and data acquisition}

Two reviewers independently assessed each eligible RCT. Differences were resolved by consensus. Funding source, experimental agent used, study phase, study duration, study outcome, sample size calculation, participant flow description, overall subject dropout, self-reported data analysis method, actual data analysis method used (determined by study reviewers), missing data proportion and missing data handling methods were assessed. ${ }^{29}$ An adequate description of study participant flow was defined as a clear description of the number of subjects who were randomised, number completing and not completing the study with reasons, and number analysed for the primary outcome. We assessed whether participant progress through the phases of parallel RCT (enrolment, allocation, follow-up and analysed) was presented in a flow diagram, as recommended. ${ }^{130}$ Definitions are given in box 1 .

Box 1 Definitions for different methods of missing data handling

\section{Definitions}

ITT analysis

- All subjects analysed after randomisation. No exclusions.

Modified ITT analysis excluded the following ${ }^{15}$ :

- Subjects who did not receive any study intervention (treatmentrelated exclusion).

- Subjects without a baseline assessment (baseline assessment exclusion).

- Subjects without a post-baseline assessment (post-baseline exclusion).

- Subjects found non-eligible after randomisation (target conditionrelated exclusion).

- Exclusion of study centre(s) due to study conduct issues (centrerelated exclusion).

Non-ITT analysis included the following:

- Performed per-protocol analysis.

- Performed a case completer analysis.

- Included exclusions not specified in the modified ITT analysis definition.

ITT, intention-to-treat. 


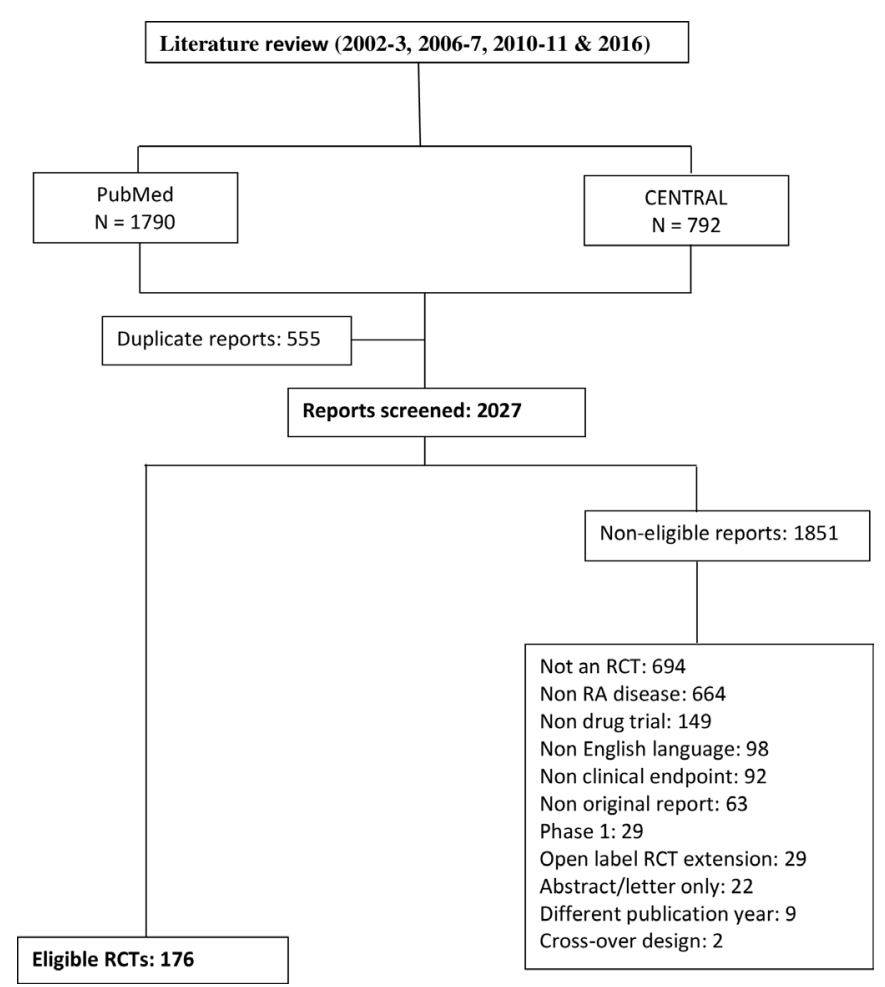

Figure 1 Study selection flow diagram. RA, rheumatoid arthritis; RCT, randomised controlled trial.

\section{Additional data for 2006 and 2016 trials}

Additional data collected for the 2006 and 2016 studies included reporting of sample size inflation to account for anticipated dropout, mechanism of missing data, missing data in each study arm, and baseline characteristics comparison of study subjects with complete and missing data, and performance of sensitivity analysis. The 2006 and 2016 RCTs were compared for data analysis, use of ITT principle and handling of missing data.

\section{Statistical analysis}

Categorical data are described as the number (per cent), and the continuous variables are described as the median (IQR). Comparison of RCT characteristics among trials using different analysis approaches and temporal changes from 2006 to 2016 was assessed using Pearson's $X^{2}$ tests, Fisher's exact test or likelihood ratio tests (for categorical variables) and Mann-Whitney U tests or Kruskal-Wallis tests (for continuous variables). SPSS V.21 was used for data analysis.

\section{RESULTS}

\section{Study characteristics}

The 2027 reports published in 7 years (2002-2003, 20062007, 2010-2011, 2016) were screened to identify 176 eligible RCTs (figure 1). These RCTs enrolled a median of 160 (IQR 62-339) patients. In terms of actual analysis: $81(46 \%)$ RCTs conducted ITT analysis, $42(23.9 \%)$ conducted mITT analysis, while $53(30.1 \%)$ conducted non-ITT analysis (27 RCTs conduced CC analysis, while analysis approach for 26 RCTs was unclear). Discordance

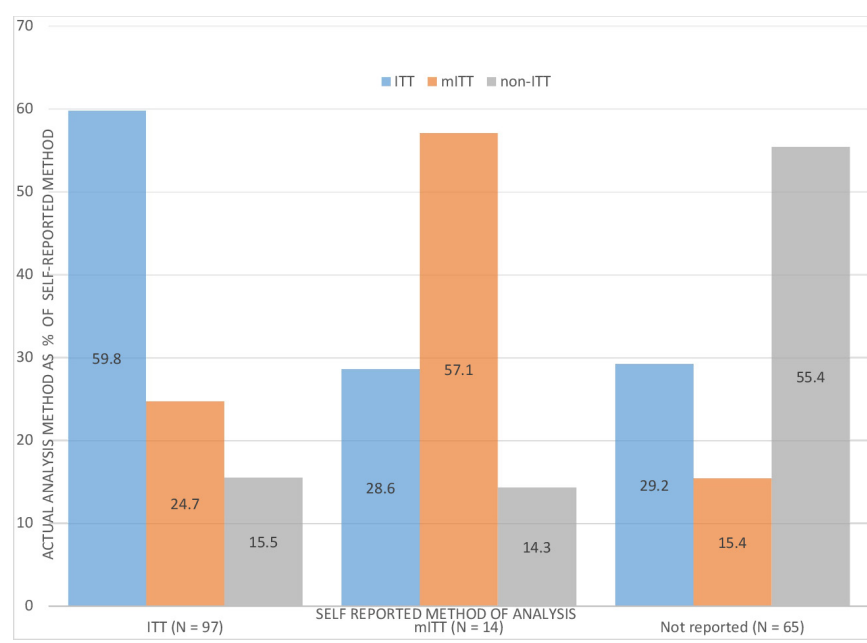

Figure 2 Discrepancy between self-reported analysis type and actual analysis performed for handling missing data. ITT, intention-to-treat; mITT, modified ITT; N, number.

was present between the reported and the actual analysis performed (figure 2). Only 58 of 97 (59.8\%) RCTs reporting an ITT analysis actually performed it. Of 42 (23.9\%) studies which performed mITT, only 8 (19.0\%) described their analysis as mITT. RCT participant flow diagram was unreported or inadequate in $66.5 \%$ RCTs: mostly resulted from non-reporting of participants analysed for the primary outcome. A median of $86 \%$ participants (IQR 74\%-91\%) remained enrolled until trial completion, although 14 RCT reports did not specify number of patients completing the trial. Fiftythree $(32.7 \%)$ and $65(40.1 \%)$ of RCTs had $>20 \%$ and 10\%-20\% missing primary outcome data, respectively. A median of $100 \%$ (IQR 97.1\%-100\%) patients were included in the efficacy analysis of primary trial outcome; 13 RCT reports did not specify number of patients analysed for the primary outcome. Most common missing data handling method was LOCF (69 $(39.2 \%))$, followed by NRI (49 (27.8\%)) and imputing missing value by interpolation (21 (11.9\%)). Only three study RCTs used preferred methods to deal with missing data for primary outcome analysis: two used linear mixed model regression, ${ }^{31}{ }^{32}$ while one used expectation-maximisation algorithm to impute missing data. ${ }^{33}$ In addition, two RCTs used equation-based methods for sensitivity analyses.

Missing data handling was not reported by 58 of 171 (33.9\%) RCTs; 5 RCTs had no missing data. Twelve RCTs using ITT analysis did not specify missing data handling method.

\section{RCT characteristics associated with analysis type}

Table 1 shows the characteristics of RCTs according to the analysis performed. Industry-funded RCTs were more likely to perform ITT or mITT than non-profit-funded RCTs. Trials reporting ITT or mITT analysis were more likely to enrol higher number of participants, be multicentre, describe sample size calculation, adequately report participant flow, describe missing data handling methods and include higher proportion of participants 
Table 1 Characteristics of the study RCTs according to the actual analysis type

\begin{tabular}{|c|c|c|c|c|}
\hline Characteristics & $\begin{array}{l}\text { ITT } \\
(\mathrm{N}=81)\end{array}$ & $\begin{array}{l}\mathrm{mITT} \\
(\mathrm{N}=42)\end{array}$ & $\begin{array}{l}\text { Non-ITT } \\
(\mathrm{N}=53)\end{array}$ & $P$ value \\
\hline \multicolumn{5}{|l|}{ Funding source } \\
\hline Industry, full or partial & $59(72.8)$ & $31(73.8)$ & $14(26.4)$ & $<0.001$ \\
\hline Non-profit or unspecified & $22(27.2)$ & $11(26.2)$ & $39(73.6)$ & \\
\hline Experimental agent & & & & 0.001 \\
\hline Traditional DMARD & $13(16.0)$ & $5(11.9)$ & $12(22.6)$ & \\
\hline Biological DMARD & $30(37)$ & $27(64.3)$ & $16(30.2)$ & \\
\hline Small molecule & $8(9.9)$ & $2(4.8)$ & $0(0.0)$ & \\
\hline Others & $30(37.0)$ & $8(19.0)$ & $25(47.2)$ & \\
\hline Study phase & & & & 0.086 \\
\hline Phase 2 & $20(24.7)$ & $8(19.0)$ & $5(9.4)$ & \\
\hline Non-phase 2/unspecified & $59(75.3)$ & $34(81.0)$ & $48(90.6)$ & \\
\hline Study centres, multiple & $63(77.8)$ & $42(100)$ & $19(35.8)$ & $<0.001$ \\
\hline Study duration, months & $6(3-12)$ & $9(3-12)$ & $6(3-12)$ & 0.481 \\
\hline Pre-study sample size calculation reported* & $42(68.9)$ & $29(85.3)$ & $24(50)$ & 0.003 \\
\hline Participant flow diagram & & & & 0.001 \\
\hline Adequate & $24(29.6)$ & $24(57.1)$ & $11(20.8)$ & \\
\hline Inadequate & $26(32.1)$ & $9(21.4)$ & $12(22.6)$ & \\
\hline Not reported & $31(38.3)$ & $9(21.4)$ & $30(56.6)$ & \\
\hline Adequate follow-up description & $63(77.8)$ & $40(95.2)$ & $33(62.3)$ & $<0.001$ \\
\hline Number of patients enrolled & $165(70-339)$ & $317(160-549)$ & $70(41-160)$ & $<0.001$ \\
\hline Percentage of patients completing RCT $\dagger$ & $85.8(70.4-89.3)$ & $82.9(76-88.9)$ & $89(72.8-92.3)$ & 0.232 \\
\hline Percentage of missing data $\dagger$ & & & & 0.341 \\
\hline $0-5$ & $8(10.5)$ & $3(7.1)$ & $6(13.6)$ & \\
\hline$>5-10$ & $10(13.2)$ & $5(11.9)$ & $12(27.3)$ & \\
\hline$>10-20$ & $32(42.1)$ & $20(47.5)$ & $13(29.5)$ & \\
\hline$>20$ & $26(34.2)$ & $14(33.3)$ & $13(29.5)$ & \\
\hline $\begin{array}{l}\text { Percentage of patients analysed for the primary } \\
\text { outcome }\end{array}$ & $100(100-100)$ & $98.7(97.1-99.6)$ & $90.8(86.3-97.7)$ & $<0.001$ \\
\hline Missing data handling method described§ & 64 (84.2) & 38 (90.5) & $11(20.8)$ & $<0.001$ \\
\hline Efficacy, positiveף & $54(69.2)$ & $35(87.5)$ & $29(63.0)$ & 0.032 \\
\hline
\end{tabular}

Values represent number (\%) for categorical variables and median (25th-75th percentile) for the numerical variables.

${ }^{*} \mathrm{~N}=143$ (33 phase $2 \mathrm{RCTs}$ excluded as they have different sample size calculation considerations).

$\dagger \mathrm{N}=162$ (five RCTs performing ITT and nine RCTs performing non-ITT analysis did not clearly report number of patients completing the trial).

$\ddagger N=163$ (13 RCTs performing non-ITT analysis did not clearly report number of patients analysed for the primary outcome).

$\S \mathrm{N}=169$ (all enrolled patients reported to complete seven RCTs with no missing data).

IPositive efficacy defined as statistically significant outcome for the primary outcome favouring the experimental intervention for superiority trials or meeting the threshold for equivalence in the non-inferiority trials. $\mathrm{N}=164,12 \mathrm{RCTs}$ were strategy trials with no intervention designated as experimental.

DMARD, disease-modifying anti-rheumatic drug; ITT, intention-to-treat; mITT, modified ITT; N, number; RCT, randomised controlled trial.

in the primary outcome analysis. However, there was no difference in proportion of participants completing the trials among the three groups. Among the mITT trials, the top two reasons for post-randomisation exclusions were no study treatment received $(\mathrm{n}=27)$ and no postbaseline assessment $(\mathrm{n}=17)$. Among non-ITT trials, the top two exclusions were study protocol deviation $(\mathrm{n}=22)$ and outcome absence at study completion $(n=7)$.

\section{Temporal trends in missing data reporting from 2006 to 2016}

These studies, 10 years apart, were compared to assess trends in missing data reporting and handling. A total of 76 studies (34 published in 2006 and 42 published in 2016) were eligible. Baseline characteristics are given in online supplemental table 1 and were similar except for more RCTs in 2016 having a placebo arm compared with 
2006. There were four RCTs of small molecules in 2016 and none in 2006.

Table 2 provides a detailed description of data reporting in 2006 and 2016 RCTs. Of the 65 non-phase 2 RCTs, only $43(66.2 \%)$ adequately reported sample size calculation. Thirteen (20.0\%) RCTs inflated sample size by median of $20 \%$ (IQR $12.5 \%-25.5 \%$ ) anticipating loss to follow-up. RCTs reporting sample size inflation numerically increased from $2006(4(12.9 \%))$ to 2016 (9 $(26.5 \%), p=0.167)$. Phase 2 trials were excluded as they have different sample size considerations. ${ }^{34}$ A significant increase in adequate participant flow description occurred between 2006 and 2016.

Self-reporting of ITT analysis declined from 2006 to 2016, while the actual analysis type was unchanged (table 2). Notably, $49(68.1 \%)$ RCTs had $>10 \%$ and 18 $(25 \%)$ had $>20 \%$ missing primary outcome data on completion. Four (5.5\%) RCTs lacked information about subjects completing the RCT. The 2016 RCTs showed a trend toward higher percentage of participants completing the trials (table 2). Nearly one-third of RCTs did not report missing outcome data handling method: unimproved from 2006 to 2016. LOCF and NRI were the most common approaches to handle missing data, although a trend in decline of LOCF use was noted. No 2006 study and two 2016 studies used the preferred missing data handling methods.

Ten of $14(17.5 \%)$ RCTs using sensitivity analysis reported results. Alternate imputation was the most used (seven RCTs) method for sensitivity testing. Sensitivity analysis usage improved from 2006 to 2016 (14.7\% vs $21.4 \%$ ). Only one trial each reported mechanism of missing data ${ }^{35}$ and baseline characteristics of trial completers and non-completers. ${ }^{36}$

Online supplemental table 2 gives the differential attrition between study arms. In 17 of 68 (25\%) RCTs which reported number of patients completing each study arm, the comparator arm had $>10 \%$ loss of patients compared with experimental arm, while three had $>10 \%$ loss in the experimental arm compared with the comparator arm.

\section{DISCUSSION}

Our review of RCTs of RA drug therapy showed that missing primary outcome data are a major issue. Reassuringly, however, almost all patients were included in the final primary outcome analysis (mostly by using SI methods for missing data). RCTs in 2016 reported the highest number of sensitivity analyses (20.5\%). The 2006 and 2016 comparisons did not show any significant improvement in the parameters of missing data reporting, handling and sensitivity analysis.

Missing data handling methods were not reported by $38.0 \%$ RCTs. This is, however, improved from a previous report where $76.5 \%$ did not do so. ${ }^{25}$ When reported, non-preferred SI methods (LOCF and NRI) were used. Limited missing data handling reporting and preference for SI methods, when $67.0 \%$ of RCTs have $>10 \%$ missing data, are concerning. To determine how much of missing data is problematic, some authors have reported a ' 5 and 20 ' rule where $<5 \%$ missing data lead to minimal bias, while $>20 \%$ pose a serious validity issue. ${ }^{37} 38$

Table 3 summarises missing data reporting in four studies, including our study. The 2005 study looked at RCT data published in high-impact journals pertaining to RA, osteoarthritis and osteoporosis. ${ }^{25}$ The 2014 paper is of RCTs published in top four medical journals irrespective of medical condition. ${ }^{24}$ The 2016 study looking at phase 3 RA RCTs published in top rheumatology and medical journals is also included. ${ }^{27}$ The latter report's RCTs may have some overlap with our study.

Misrepresentation of ITT analysis was common with only 59.8\% of self-reported ITT being true ITT analysis. Overall, $46.0 \%$ actually performed an ITT analysis, which is slightly higher as compared with some other reports of $37 \%-40 \%^{24} 26$ and improved from older RA studies at $7.4 \%{ }^{25} \mathrm{mITT}$ analysis excludes subjects which violate the ITT principles. However, the number of patients actually analysed for the primary outcome was high: $100 \%$ for ITT (as expected) and $98.8 \%$ for mITT. Thus, the absolute patient exclusions in mITT RCTs are small. Exclusion of such subjects, especially with small numbers, does not always violate the ITT principle. ${ }^{915273940}$ Recently, the treatment effect estimation in RA trials was shown to be similar between mITT and ITT analysis. ${ }^{39}$

For the 2006 and 2016 studies, only 20\% adjusted the sample size for dropouts despite a quarter having $>20 \%$ missing outcome data. Sample size inflation accounts for dropout data loss but it does not remedy missing data and any differences between the observed and missed data. ${ }^{16}$ Differential attrition can be a source of bias in RCTs. ${ }^{37}$ In our analysis, $>10 \%$ attrition in the placebo or comparator arm versus the experimental arm was seen in $25 \%$ of trials which may artificially increase treatment effect in favour of experimental intervention if using SI. The use of SI in differential attrition biases results. ${ }^{41} \mathrm{~A}$ sensitivity analysis is required to assess the impact of such possible bias, but was performed in $<20 \%$ trials. Impact of differential attrition in RA is unexplored. A small study of musculoskeletal RCTs found differential attrition rates ranging from $1 \%$ to $14 \%{ }^{42}$ without impacting the results. The authors suggested reporting of baseline characteristics of both the analysed and randomised subjects.

Only one study reported a missing data mechanism. ${ }^{35}$ Another review reported this number at $7.8 \%$ RCTs. ${ }^{27}$ Three missing data mechanisms exist: missing completely at random (MCAR), missing at random (MAR) and missing not at random (MNAR) and are discussed elsewhere. ${ }^{43} 44$ It is important to obtain data on study completers and the non-completers. It is possible to distinguish between MCAR and MAR by studying the available data. If the completers and the non-completers differ (for example, if older participants have more missing data than younger participants, the data are not MCAR but possibly MAR). It is not possible to distinguish between MAR and MNAR by studying the available data. ${ }^{24}$ Only one RCT in our 
Table 2 Comparison of data reporting including missing data and its handling in 2006 and 2016 RCTs

\begin{tabular}{|c|c|c|c|c|}
\hline \multirow[b]{2}{*}{ Characteristics } & \multirow{2}{*}{$\begin{array}{l}\text { All } \\
(\mathrm{N}=76)\end{array}$} & \multicolumn{2}{|l|}{ Study year } & \multirow[b]{2}{*}{$P$ value } \\
\hline & & $2006(\mathrm{~N}=34)$ & 2016 (N=42) & \\
\hline Total patients & & & & 0.913 \\
\hline Median (IQR) & $162(74-326)$ & $163(53-367)$ & $159(79-311)$ & \\
\hline Range & $21-1404$ & $21-1093$ & $43-1404$ & \\
\hline Patient per cent completing study* & & & & 0.089 \\
\hline Median (IQR) & $86.4(79.5-91.6)$ & $84.5(72.8-91.8)$ & $87.9(81.7-91.6)$ & \\
\hline Range & $38.5-100$ & $40.9-95.8$ & $38-5-100$ & \\
\hline $\begin{array}{l}\text { Patient per cent analysed for primary } \\
\text { outcome† }\end{array}$ & & & & 0.962 \\
\hline Median (IQR) & $99.7(97.1-100)$ & $99.5(97.3-100)$ & $99.7(97-100)$ & \\
\hline Range & $38.5-100$ & $85.8-100$ & $38.5-100$ & \\
\hline Patient in experiment arm(s) & & & & 0.81 \\
\hline Median (IQR) & $100(40-217)$ & $99(29-252)$ & $103(40-177)$ & \\
\hline Range & $11-1286$ & $11-751$ & $19-1286$ & \\
\hline $\begin{array}{l}\text { Patient per cent completing study in } \\
\text { experimental arm(s) } \neq\end{array}$ & & & & 0.405 \\
\hline Median (IQR) & $87.7(81.3-93.1)$ & $86.4(81.7-93)$ & $88.6(80.7-94.4)$ & \\
\hline Range & $37.5-100$ & $45.2-100$ & $37.5-100$ & \\
\hline $\begin{array}{l}\text { Patient per cent analysed for primary } \\
\text { outcome in experimental arm(s)§ }\end{array}$ & & & & 0.721 \\
\hline Median (IQR) & $100(99.6-100)$ & $100(99.3-100)$ & $100(99.6-100)$ & \\
\hline Range & $37.5-100$ & $88.6-100$ & $37.5-100$ & \\
\hline Patient in comparator arm & & & & 0.983 \\
\hline Median (IQR) & $56(30-132)$ & $68(25-141)$ & $53(31-121)$ & \\
\hline Range & $10-532$ & $10-531$ & $15-532$ & \\
\hline $\begin{array}{l}\text { Patient per cent completing study in } \\
\text { comparator arm }\end{array}$ & & & & 0.1 \\
\hline Median (IQR) & $84.4(73-91.5)$ & $81.9(64.6-90.6)$ & $84.9(78.1-93.5)$ & \\
\hline Range & $23.9-100$ & $30-93.7$ & $23.9-100$ & \\
\hline $\begin{array}{l}\text { Patient per cent analysed for primary } \\
\text { outcome in comparator arm }\end{array}$ & & & & 0.507 \\
\hline Median (IQR) & $100(97.2-100)$ & $100(96.1-100)$ & $100(97.6-100)$ & \\
\hline Range & $39.5-100$ & $84.5-100$ & $39.5-100$ & \\
\hline $\begin{array}{l}\text { Adequate sample size calculation } \\
\text { descriptiont† }\end{array}$ & $43(66.2)$ & 19 (61.3) & $24(70.6)$ & 0.429 \\
\hline $\begin{array}{l}\text { Sample size inflation anticipating follow-up } \\
\text { losst† }\end{array}$ & $13(20.0)$ & $4(12.9)$ & $9(26.5)$ & 0.167 \\
\hline Adequate follow-up description & $64(84.2)$ & $28(82.4)$ & $36(85.7)$ & 0.689 \\
\hline Flow diagram for patient follow-up & & & & 0.016 \\
\hline Adequate & $31(40.8)$ & $17(50.0)$ & $14(33.3)$ & \\
\hline Inadequate & $18(23.7)$ & $3(8.8)$ & $15(35.7)$ & \\
\hline Not reported & $27(35.5)$ & $14(41.2)$ & $13(31.0)$ & \\
\hline Amount of missing outcome data* & & & & 0.042 \\
\hline$<5 \%$ & $10(13.9)$ & $1(3.1)$ & $9(22.5)$ & \\
\hline $5.1 \%-10 \%$ & $13(18.1)$ & $8(25.0)$ & $5(12.5)$ & \\
\hline $10.1 \%-20 \%$ & $31(43.1)$ & $13(40.6)$ & $18(45.0)$ & \\
\hline$>20 \%$ & $18(25)$ & $10(31.3)$ & $8(20.0)$ & \\
\hline
\end{tabular}


Table 2 Continued

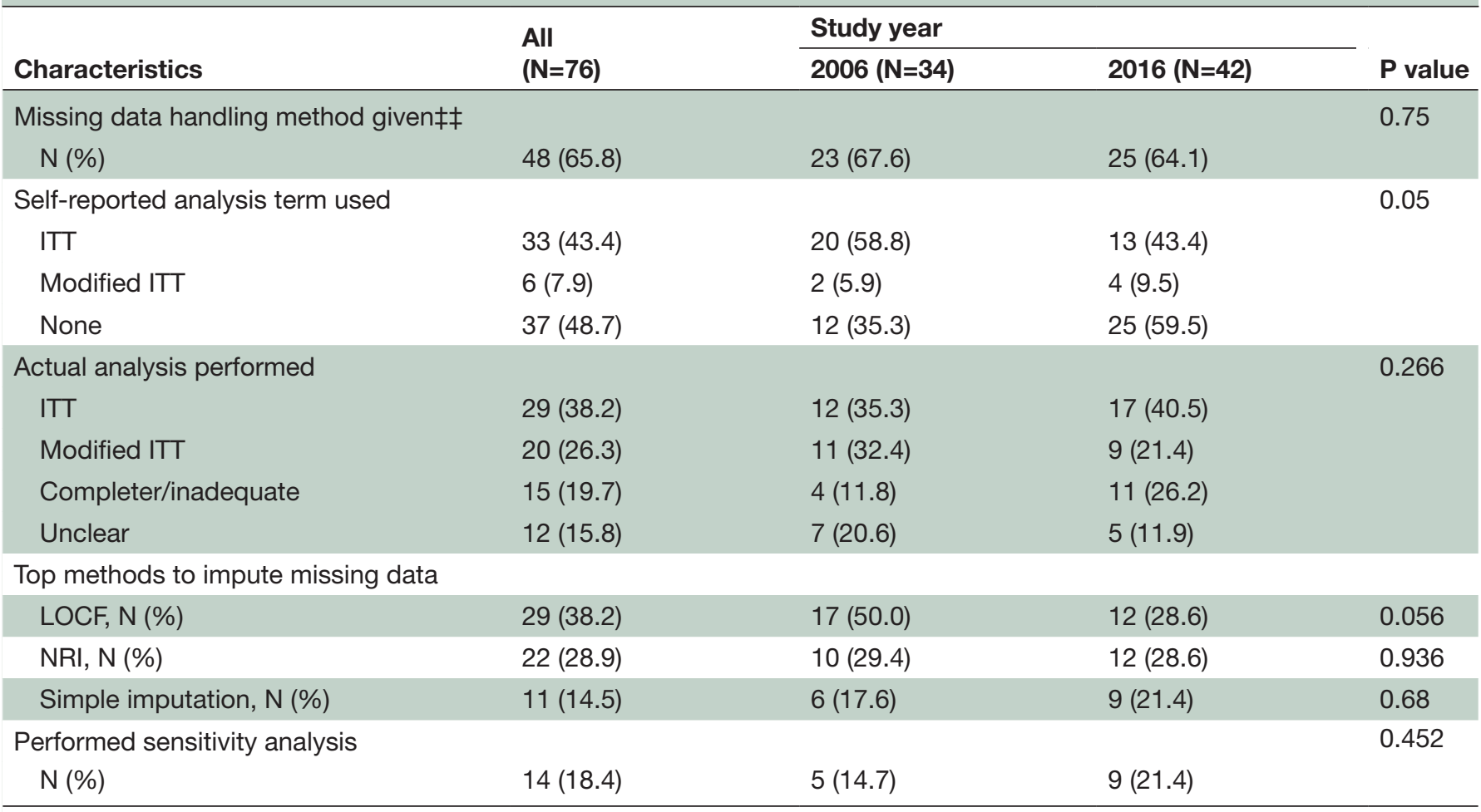

${ }^{*} \mathrm{~N}=72$, number of patients completing the trial unclear for four RCTs.

$\dagger \mathrm{N}=73$, number of patients included in primary outcome analysis unclear for three RCTs.

$\ddagger \mathrm{N}=69$, number of patients in experimental arm(s) completing the trial unclear for seven RCTs.

$\S \mathrm{N}=70$, number of patients in experimental arm(s) included in primary outcome analysis unclear for six RCTs.

ๆN=75, one RCT had three arms with different doses of experimental intervention, no comparator group.

${ }^{* *} \mathrm{~N}=68$, number of patients in comparator arm completing the trial unclear for seven RCTs and one RCT did not have a comparator group.

$\dagger † \mathrm{~N}=65$, excluded 11 phase $2 \mathrm{RCTs}$ as they have different sample size calculation considerations.

$\ddagger \ddagger \mathrm{N}=73$, all enrolled patients reported to complete three RCTs with no missing data.

ITT, intention-to-treat; LOCF, last observation carried forward; N, number; NRI, non-response imputation; RCTs, randomised controlled trials.

Table 3 Trend of salient characteristics over time in RA studies and comparison with a study from top four medical journals

\begin{tabular}{|c|c|c|c|c|c|}
\hline \multirow[b]{2}{*}{ Characteristic } & \multicolumn{5}{|c|}{ Time period of included studies/(reference) (publication year) } \\
\hline & $\begin{array}{l}1994-2003^{25} \\
(2005)\end{array}$ & $\begin{array}{l}2006 \\
\text { (Current) }\end{array}$ & $\begin{array}{l}2016 \\
\text { (Current) }\end{array}$ & $\begin{array}{l}2013^{* 24} \\
(2014)\end{array}$ & $\begin{array}{l}2008-2013^{* 27} \\
(2016)\end{array}$ \\
\hline Overall ITT analysis performed, \% & 7.4 & 35.3 & 38.1 & 40.0 & $\mathrm{NA} \dagger$ \\
\hline Overall PP analysis performed, \% & 59.3 & 11.7 & 28.5 & NA & NA \\
\hline Preferred missing data handling methods used, \% & 1.2 & 2.7 & 4.9 & 27.3 & 1.9 \\
\hline Dropout in each study arm given, \% & 69.0 & 82.3 & 85.7 & 97.0 & NA \\
\hline Missing data handling given, \% & 23.5 & 61.1 & 48.8 & 36.0 & 94.1 \\
\hline LOCF used to handle missing datał, \% & 52.6 & 41.7 & 22.7 & 12.0 & 56.8 \\
\hline Missing mechanism given, $\%$ & NA & 2.9 & 0.0 & NA & 7.8 \\
\hline Sensitivity analysis performed, \% & 11.1 & 13.9 & 20.5 & 37.0 & 27.0 \\
\hline Comparison of completers/non-completers, \% & 16.7 & 0.0 & 2.3 & 11.6 & NA \\
\hline
\end{tabular}

${ }^{*}$ Studies involving top medical journals.

†86\% stated that ITT was used but no information on those that actually performed ITT.

fLOCF was the most common method used across all years for imputation except 2016 (lbrahim et al) where NRI was most frequent (74.5\%) but NRI and LOCF were used simultaneously as well; in the 1994-2003 and 2013 studies, complete case analysis (59.3\% and $45.0 \%$, respectively) was the most common method to handle missing data.

ITT, intention-to-treat; LOCF, last observation carried forward; NA, not available; PP, per-protocol; RA, rheumatoid arthritis. 
analysis reported data on completers and dropouts. ${ }^{36}$ In one study, this was $11.6 \%{ }^{24}$ and $16.7 \%$ in older RA trials. ${ }^{25}$ Generally, MAR is a reasonable starting point. ${ }^{1621}$ In our study, LOCF was the most common method $(53.7 \%)$ to handle missing data in the 95 RCTs that used ITT or mITT. This is different from a prior report of RA trials where NRI was the most common $(75 \%)$ imputation method. ${ }^{27}$ The US Food and Drug Administration as well as the European Medicines Agency used to recommend LOCF method, assuming that it could provide conservative effect estimates. This may have contributed to its widespread use. However, it generally introduces bias even if data are missing completely at random. ${ }^{45}$ In an RCT with longitudinal data, it is generally recommended to use a mixed model analysis excluding any systematic treatment effect at baseline. This is called 'constrained analysis' ${ }^{46}$ It is also recommended to adjust for baseline value of the outcome variable. ${ }^{47}$ In particular, this is a good way to handle missing data in an RCT with longitudinal data. ${ }^{48}$ None of our study RCTs used this approach.

A sensitivity analysis to test a range of plausible mechanisms responsible for the missing data is generally recommended. ${ }^{7}$ A sensitivity analysis should be performed with a different mechanism of missingness. ${ }^{44}$ Agreement of different sensitivity analyses is reassuring. Sensitivity analysis was reported by $17.5 \%$ RCTs in our review. These numbers are similar (21\%-27\%) for recent studies reviewing sensitivity analysis in RA trials ${ }^{26}$ but lower than numbers $(37 \%$ and $27 \%$ ) from top medical journals. $^{2427}$

Our study has limitations. The RCTs in our study were time and language restricted. Results may not be applicable to non-RA trials. Some of the deficiencies may result from poor reporting (not mentioning relevant details) and not necessarily poor execution. ${ }^{49}$ On the other hand, we have looked at a large number of trials over several years. We have compared the trend in reporting at two time points separated by a decade. We have not restricted our analysis to high-impact journals increasing representation.

Major gaps exist between statistical analysis recommendations and actual reporting. Many papers and recommendations have addressed these deficiencies from a statistical perspective. ${ }^{1623445051}$ These issues are seemingly better handled in papers published in high-ranking journals signifying greater attention to missing data handling during peer review might improve such reporting.

In conclusion, our study has found significant shortcomings in the reporting and handling of missing data in RA trials: there is wide variability in reporting and performance of ITT analysis, SI methods remain popular despite shortcomings, sensitivity analysis usage is low, there is inadequate explicit reporting of participants with missing outcomes and methods used to handle them, baseline comparisons of completers versus dropouts are deficient. Lack of a significant trend in improved reporting 10 years apart remains a major concern.
Author affiliations

${ }^{1}$ Rheumatology Section, CHI St Vincent Little Rock Diagnostic Clinic, Little Rock, Arkansas, USA

${ }^{2}$ Division of Rheumatology, Loma Linda University, Loma Linda, California, USA ${ }^{3}$ Divison of Rheumatology, Department of Internal Medicine, Mayo Clinic, Scottsdale, Arizona, USA

Presented at

This study has been partially presented as a poster at the 2017 American College of Rheumatology annual meeting as a poster and as an oral presentation at the 2018 American College of Rheumatology annual meeting. References are the following: 2017 conference reference: Aslam F, Torralba K, Khan NA. Handling of Missing Data, Protocol Violation and Performance of Intention-to-Treat Analysis in the Randomized Controlled Trials of Drug Therapy of Rheumatoid Arthritis (abstract). Arthritis Rheumatol. 2017; 69 (suppl 10). https://acrabstracts.org/ abstract/handling-of-missing-data-protocol-violation-and-performance-ofintention-to-treat-analysis-in-the-randomized-controlled-trials-of-drug-therapyof-rheumatoid-arthritis/. Accessed 11 March 2020. 2018 conference reference: Aslam F, Torralba K, Khan NA. Comparison of Missing Data Reporting and Handling in Randomized Controlled Trials of Rheumatoid Arthritis Drug Therapy: A Snapshot Ten Years Apart (abstract). Arthritis Rheumatol. 2018; 70 (suppl 10). https:// acrabstracts.org/abstract/comparison-of-missing-data-reporting-and-handlingin-randomized-controlled-trials-of-rheumatoid-arthritis-drug-therapy-a-snapshotten-years-apart/https://acrabstracts.org/abstract/comparison-of-missing-datareporting-and-handling-in-randomized-controlled-trials-of-rheumatoid-arthritisdrug-therapy-a-snapshot-ten-years-apart/. Accessed 11 March 2020.

\section{Twitter Fawad Aslam @faslammd}

Contributors All authors contributed to the drafting, writing, editing and review of the manuscript. NAK and KDT conceived the idea. FA and NAK collected the data. NAK ran the analysis.

Funding The authors have not declared a specific grant for this research from any funding agency in the public, commercial or not-for-profit sectors.

Competing interests None declared.

Patient consent for publication Not required.

Provenance and peer review Not commissioned; externally peer reviewed. Data availability statement Data available upon request.

Open access This is an open access article distributed in accordance with the Creative Commons Attribution Non Commercial (CC BY-NC 4.0) license, which permits others to distribute, remix, adapt, build upon this work non-commercially, and license their derivative works on different terms, provided the original work is properly cited, appropriate credit is given, any changes made indicated, and the use is non-commercial. See: http://creativecommons.org/licenses/by-nc/4.0/.

ORCID iD

Fawad Aslam http://orcid.org/0000-0001-6016-6123

\section{REFERENCES}

1 Moher D, Hopewell S, Schulz KF, et al. Consort 2010 explanation and elaboration: updated guidelines for reporting parallel group randomised trials. BMJ 2010;340:c869.

2 White IR, Horton NJ, Carpenter J, et al. Strategy for intention to treat analysis in randomised trials with missing outcome data. BMJ 2011;342:d40.

3 Liao JM, Stack CB. Annals understanding clinical research: implications of missing data due to dropout. Ann Intern Med 2017;166:596-8.

4 Marston L, Sedgwick P. Randomised controlled trials: missing data. BMJ 2014;349:94656.

5 Sedgwick P. Intention to treat analysis versus per protocol analysis of trial data. BMJ 2015;350:h681.

6 Horwitz RI, Viscoli CM, Berkman L, et al. Treatment adherence and risk of death after a myocardial infarction. Lancet 1990;336:542-5.

7 DeMets DL, Cook T. Challenges of Non-Intention-to-Treat analyses. JAMA 2019;321:145-6.

8 Sackett DL, Gent M. Controversy in counting and attributing events in clinical trials. N Engl J Med 1979;301:1410-2.

9 Kunz R, Guyatt G. Which patients to include in the analysis? Transfusion 2006;46:881-4.

10 Gupta SK. Intention-To-Treat concept: a review. Perspect Clin Res 2011;2:109-12. 
11 Hollis $\mathrm{S}$, Campbell $\mathrm{F}$. What is meant by intention to treat analysis? Survey of published randomised controlled trials. BMJ 1999;319:670-4.

12 Gravel J, Opatrny L, Shapiro S. The intention-to-treat approach in randomized controlled trials: are authors saying what they do and doing what they say? Clinical Trials 2007;4:350-6.

13 Herman A, Botser IB, Tenenbaum S, et al. Intention-To-Treat analysis and accounting for missing data in orthopaedic randomized clinical trials. J Bone Joint Surg Am 2009;91:2137-43.

14 Montedori A, Bonacini MI, Casazza G, et al. Modified versus standard intention-to-treat reporting: are there differences in methodological quality, sponsorship, and findings in randomized trials? A cross-sectional study. Trials 2011;12:58.

15 Abraha I, Montedori A. Modified intention to treat reporting in randomised controlled trials: systematic review. BMJ 2010;340:c2697.

16 Little RJ, D'Agostino R, Cohen ML, et al. The prevention and treatment of missing data in clinical trials. N Engl J Med 2012;367:1355-60.

17 O'Neill RT, Temple R. The prevention and treatment of missing data in clinical trials: an FDA perspective on the importance of dealing with it. Clin Pharmacol Ther 2012;91:550-4.

18 Ware $\mathrm{JH}$. Interpreting incomplete data in studies of diet and weight loss. N Engl J Med 2003;348:2136-7.

19 Lagakos SW. Time-to-event analyses for long-term treatments--the APPROVe trial. N Engl J Med 2006;355:113-7.

20 Molnar FJ, Man-Son-Hing M, Hutton B, et al. Have last-observationcarried-forward analyses caused us to favour more toxic dementia therapies over less toxic alternatives? A systematic review. Open Med 2009;3:e31-50.

21 Thabane L, Mbuagbaw L, Zhang S, et al. A tutorial on sensitivity analyses in clinical trials: the what, why, when and how. BMC Med Res Methodol 2013;13:92.

22 Altman DG. Missing outcomes in randomized trials: addressing the dilemma. Open Med 2009;3:e51-3.

23 Cro S, Morris TP, Kenward MG, et al. Sensitivity analysis for clinical trials with missing continuous outcome data using controlled multiple imputation: a practical guide. Stat Med 2020;39:2815-42.

24 Bell ML, Fiero M, Horton NJ, et al. Handling missing data in RCTs; a review of the top medical journals. BMC Med Res Methodol 2014;14:118

25 Baron G, Boutron I, Giraudeau B, et al. Violation of the intent-totreat principle and rate of missing data in superiority trials assessing structural outcomes in rheumatic diseases. Arthritis Rheum 2005;52:1858-65.

26 Joseph R, Sim J, Ogollah R, et al. A systematic review finds variable use of the intention-to-treat principle in musculoskeletal randomized controlled trials with missing data. J Clin Epidemiol 2015;68:15-24.

27 Ibrahim F, Tom BDM, Scott DL, et al. A systematic review of randomised controlled trials in rheumatoid arthritis: the reporting and handling of missing data in composite outcomes. Trials 2016;17:272.

28 Evans SR, Bigelow R, Chuang-Stein C, et al. Presenting risks and benefits: helping the data monitoring Committee do its job. Ann Intern Med 2020;172:119-25.

29 Khan NA, Lombeida JI, Singh M, et al. Association of industry funding with the outcome and quality of randomized controlled trials of drug therapy for rheumatoid arthritis. Arthritis Rheum 2012;64:2059-67.

30 Egger M, Jüni P, Bartlett C, et al. Value of flow diagrams in reports of randomized controlled trials. JAMA 2001;285:1996-9.

31 Goekoop-Ruiterman YPM, de Vries-Bouwstra JK, Allaart CF, et al. Comparison of treatment strategies in early rheumatoid arthritis: a randomized trial. Ann Intern Med 2007;146:406-15.
32 Fautrel B, Pham T, Alfaiate T, et al. Step-Down strategy of spacing TNF-blocker injections for established rheumatoid arthritis in remission: results of the multicentre non-inferiority randomised open-label controlled trial (STRASS: spacing of TNFblocker injections in rheumatoid arthritis study). Ann Rheum Dis 2016;75:59-67.

33 Wilkinson TJ, Lemmey AB, Jones JG, et al. Can creatine supplementation improve body composition and objective physical function in rheumatoid arthritis patients? A randomized controlled trial. Arthritis Care Res 2016;68:729-37.

34 Stallard N. Optimal sample sizes for phase II clinical trials and pilot studies. Stat Med 2012;31:1031-42.

35 Kremer JM, Genant HK, Moreland LW, et al. Effects of abatacept in patients with methotrexate-resistant active rheumatoid arthritis: a randomized trial. Ann Intern Med 2006;144:865-76.

36 Rajaei E, Mowla K, Ghorbani A, et al. The effect of omega-3 fatty acids in patients with active rheumatoid arthritis receiving DMARDs therapy: double-blind randomized controlled trial. Glob J Health Sci 2015;8:18-25.

37 Schulz KF, Grimes DA. Sample size slippages in randomised trials: exclusions and the lost and wayward. Lancet 2002;359:781-5.

38 Straus SE, Glasziou P, Richardson SW. Prognosis. Evidence-based medicine : how to practice and teach ebm. Edinburgh: Elsevier Health Sciences, 2019: 190

39 Dossing A, Tarp S, Furst DE, et al. Modified intention-to-treat analysis did not bias trial results. J Clin Epidemiol 2016;72:66-74

40 Fergusson D, Aaron SD, Guyatt G, et al. Post-Randomisation exclusions: the intention to treat principle and excluding patients from analysis. BMJ 2002;325:652-4.

41 Bell ML, Kenward MG, Fairclough DL, et al. Differential dropout and bias in randomised controlled trials: when it matters and when it may not. BMJ 2013;346:e8668.

42 Hewitt CE, Kumaravel B, Dumville JC, et al. Assessing the impact of attrition in randomized controlled trials. $J$ Clin Epidemiol 2010;63:1264-70.

43 Dziura JD, Post LA, Zhao Q, et al. Strategies for dealing with missing data in clinical trials: from design to analysis. Yale $\mathrm{J} \mathrm{Biol} \mathrm{Med}$ 2013;86:343-58.

44 National Research Council (US) Panel on Handling Missing Data in Clinical Trials. The prevention and treatment of missing data in clinical trials, 2010. Available: https://www.ncbi.nlm.nih.gov/books/ NBK209904/ 10.17226/12955 [Accessed Mar 2021].

45 Lydersen S. Last observation carried forward. Tidsskr Nor Laegeforen 2019;139. doi:10.4045/tidsskr.19.0061. [Epub ahead of print: 28 May 2019].

46 Coffman CJ, Edelman D, Woolson RF. To condition or not condition? Analysing 'change' in longitudinal randomised controlled trials. $B M J$ Open 2016;6:e013096.

$47 \mathrm{~J} \mathrm{~T}, \mathrm{~L} \mathrm{~B}, \mathrm{~T}$ H, et al. Different ways to estimate treatment effects in randomised controlled trials. Contemp Clin Trials Commun 2018;10:80-5.

48 Twisk JW, Rijnhart JJ, Hoekstra T, et al. Intention-To-Treat analysis when only a baseline value is available. Contemp Clin Trials Commun 2020;20:100684.

49 Soares HP, Daniels S, Kumar A, et al. Bad reporting does not mean bad methods for randomised trials: observational study of randomised controlled trials performed by the radiation therapy Oncology Group. BMJ 2004;328:22-4.

50 Altman DG, Schulz KF, Moher D, et al. The revised consort statement for reporting randomized trials: explanation and elaboration. Ann Intern Med 2001;134:663-94.

51 Moher D, Hopewell S, Schulz KF, et al. Consort 2010 explanation and elaboration: updated guidelines for reporting parallel group randomised trials. Int J Surg 2012;10:28-55. 